\title{
HARMONIC ANALYSIS OF HARMONIC FUNCTIONS IN THE PLANE
}

\author{
L. A. RUBEL
}

\begin{abstract}
A continuous function on the complex plane is harmonic if and only if the span of its compositions with entire functions is not dense in the space of continuous functions in the topology of uniform convergence on compact sets.
\end{abstract}

Let $\mathbf{C}$ be the complex plane and let $E$ be the space of all entire functions. We remark that these functions $\varphi$ (and their conjugates) are precisely those functions for which $f \circ \varphi$ is harmonic whenever $f$ is. Let $C(\mathbf{C})$ be the space of all complex-valued continuous functions on $\mathbf{C}$ in the topology of uniform convergence on compact sets. The next definition is motivated by the theory of mean-periodic functions (see [2]).

Definition. A variety is a closed linear subspace $V$ of $C(\mathbf{C})$ that is invariant under composition with all entire functions.

This means that if $f \in V$ and $\varphi \in E$, then $f \circ \varphi \in V$. The problems of spectral analysis and spectral synthesis are completely resolved by the following result.

THE SIX-VARIETIES THEOREM. There are exactly six varieties:

1. $\{0\}$,

2. $\mathbf{C}$ (the constant functions),

3. $E$,

4. $\bar{E}$ (the conjugates of entire functions),

5. $H$ (all harmonic functions),

6. $C(\mathbf{C})$.

Corollary 1. If $f \in C(\mathbf{C})$ is not harmonic, then the span of its compositions with all entire functions is dense in $C(\mathbf{C})$.

This corollary may be regarded as an approximation theorem. An analogue, for the operator $\partial^{2} / \partial x \partial y$ instead of the Laplacian, was proved in [1].

COROllary 2. If $f$ is a nonconstant entire function, then the span of its compositions with all entire functions is dense in $E$.

In point of fact, the theorem amounts to little more than these two corollaries, so we just give their proofs, from which a proof of the theorem may be easily devised.

Presented to the Society, April 12, 1975 under the title Harmonic analysis of harmonic functions; received by the editors November 11, 1974.

AMS (MOS) subject classifications (1970). Primary 31A05, 43A45.

(c) American Mathematical Society 1976 
Proof of Corollary 1. We first suppose that $f \in C^{2}(\mathbf{C})$, i.e. that $f$ has continuous second partial derivatives. Now if the span of the compositions of $f$ with entire functions is not dense in $C(\mathbf{C})$, then there exists a Borel measure $\mu$ of compact support such that

$$
\int f(\varphi(z)) d \mu(z)=0 \quad \forall \varphi \in E .
$$

Now for any $w \in \mathbf{C}$, we also have

$$
A(w)=\int f(\varphi(z)) d \mu(z-w)=0 \quad \forall \varphi \in E,
$$

so that on integrating $A(w) d \nu(w)$, where $\nu$ is absolutely continuous with respect to planar Lebesgue measure, we get $\int f(\varphi(z)) d(\mu * \nu)(z)=0$. So without loss of generality, we may suppose that $\mu$ in (1) is absolutely continuous. We rewrite (1) as $\int f\left(\sum a_{k} z^{k}\right) d \mu(z)^{\star}=0$ for every everywhere convergent power series. Now comes the main idea. Taking $\partial^{2} / \partial a_{m} \partial \bar{a}_{n}$, we get

$$
\int f_{z \bar{z}}\left(\sum a_{k} z^{k}\right) z^{m} \bar{z}^{n} d \mu(z)=0
$$

so that for all polynomials $P(z, \bar{z})$ we have

$$
\int f_{z \bar{z}}\left(\sum a_{k} z^{k}\right) P(z, \bar{z}) d \mu(z)=0 .
$$

By the Stone-Weierstrass theorem, we conclude that for each continuous function $g$,

$$
\int f_{z \bar{z}}(\varphi(z)) g(z) d \mu(z)=0 \quad \forall \varphi \in E .
$$

But then (2) holds for any bounded measurable function $g$ on the support of $\mu$, and we conclude that $f_{z \bar{z}}(\varphi(z))=0$ a.e. $\mu$.

Since $\mu$ is absolutely continuous, the support of $\mu$ has a point of density $z_{0}$. Then $f_{z \bar{z}}(\varphi(z))$ has a zero in each neighborhood of $z_{0}$ so that $f_{\bar{z} \bar{z}}\left(\varphi\left(z_{0}\right)\right)=0$ and consequently $f_{z \bar{z}} \equiv 0$. But $4 f_{z \bar{z}}$ is the Laplacian of $f$ and thus $f$ is harmonic.

For the general case where $f \notin C^{2}(\mathbf{C})$, we convolve $f$ with an approximate identity that is twice continuously differentiable. This argument is standard, and we omit the details.

Proof of Corollary 2. In a similar circle of ideas, we suppose $\int f \circ \varphi d \mu$ $=0$ for all $\varphi \in E$ and we must prove that $\int g d \mu=0$ for all $g \in E$. Consider $\int f\left(a e^{\lambda g(z)}\right) d \mu(z)=0$ where $g \in E$ is arbitrary. Take $d /\left.d \lambda\right|_{\lambda=0}$ to get $a f^{\prime}(a) \int g(z) d \mu(z)=0$, and the conclusion follows since $f$ is not constant.

REMARKS. The analogue of Corollary 1 is easily verified for a disc. It might be true for transitive regions $G$, that is, regions for which, for every $z_{0}, w_{0}$ $\in G$, there exists an analytic function $\varphi: G \rightarrow G$ such that $\varphi\left(z_{0}\right)=w_{0}$ and $\varphi^{\prime}\left(z_{0}\right) \neq 0$. But the analogue is false for $G=\mathbf{C} \backslash\{-1,1\}$, for on applying Picard's Great Theorem, we see that there are too few analytic functions $\varphi: G \rightarrow G$. Similarly, the analogue of our result for other dimensions than $n=2$ fails because there are too few maps that preserve harmonicity. 


\section{REFERENCES}

1. L. A. Rubel and A. L. Shields, Hyperbolic mean automorphic functions, Invent. Math. 4 (1967), 294-298. MR 37 \# 1975.

2. L. Schwartz, Théorie générale des fonctions moyenne-périodiques, Ann. of Math. (2) 48 (1947), 857-929. MR 9, 428.

Department of Mathematics, University of Illinois at Urbana-Champaign, Urbana, ILLINOIS 61801 